\title{
Quality of Life in Schizophrenia and Bipolar Affective Disorder
}

\author{
Pratima $^{1 *}$
}

\section{ABSTRACT}

Family caregivers of persons with bipolar disorder and schizophrenia experience high level of burden and compromised quality of life. A considerable amount of burden on the caregivers often leads to display of certain attitudes towards persons with severe mental illness called expressed emotion, which then leads to poor quality of patients as well. Although numerous studies dealing with these issues separately are present, but studies dealing with relationship, using mixed methodology, among these issues are scarce. The aim of the present study was to understand how actually the construct of quality of life in different demographic conditions affect life conditions of schizophrenic and bipolar patients and determining relapse. The present study was designed mainly to assess the quality of life on patients and the families of a particular group of patients namely those with schizophrenia and bipolar disorder. The objectives if the present research were to study: (i) the quality of life of patients with Schizophrenia and Bipolar Affective disorder. (ii) the quality of life of caregivers of patients with Schizophrenia and Bipolar Affective disorder. Patients with disorders such as schizophrenia and bipolar affective disorder are more likely to relapse when there is high expressed emotion present in their living environment. The stress from the remarks and attitudes of the family is overwhelming because they feel like the cause of the problems. The patient then falls into the cycle of relapse. The only way to escape this vortex for the family is to go through therapy together to prevent the relapse. But before that it becomes necessary to understand that what is the reason behind such attitude towards a family member who is mentally ill, what is the cause of burden and what all changes the caregivers' and the patients' quality of life come across.

Keywords: Quality, Life, Schizophrenia, Bipolar Affective

In the scientific literature, the term quality of life is used with many different meanings and refers to a loosely related body of work on psychological well- being, social and emotional functioning, functional performance, life satisfaction, social support etc. The study of quality of life and the focus on patient's subjective sense of well- being is a fairly new phenomenon that has attracted professional attention only within the past two decade. Between 1850 and 1950, medicine was dominated by the quest for cures; treating chronic illness as well as helping patients manages long-term impairment received less attention. Issues of life quality that cannot

\footnotetext{
${ }^{1}$ Assistant Professor, Amity Institute of Psychology and Allied Science, Amity University, Noida, U.P *Responding Author

(C) 2015 I Pratima; licensee IJIP. This is an Open Access Research distributed under the terms of the Creative Commons Attribution License (http://creativecommons.org/licenses/by/2.0), which permits unrestricted use, distribution, and reproduction in any Medium, provided the original work is properly cited.
} 


\section{Quality of Life in Schizophrenia and Bipolar Affective Disorder}

be eliminated must be managed and the treatment goal becomes maintaining maximum functioning and a meaningful existence or quality of life. With the increasing awareness of the multidimensionality of the treatment outcome and the importance of patient satisfaction in health care, the construct of quality of life has become an important area of investigation. Especially the interest in quality of life in psychiatry has been stimulated by the deinstitutionalization of psychiatric patients as well as a parallel interest in understanding the scope of their daily lives. Studies also focused on the quality of life rather than cure of illness in psychiatric patients living in community (Brown et. al. 1998). Doyle et. al. (1999) also identified quality of life as an important measure of impact of schizophrenia and its respective treatment.

\section{Quality of Life and Mental Illness}

Mental and behavioral disorders cause massive disruption in the lives of those who are affected and on their families. Quality of life of persons with severe mental illness would be substantial and sustained due to its negative impact on the illness. Studies also showed that individuals with severe mental illness living in long term institutional set up had poorer quality of life than those living in the community (Olweny, 1992). One of the study demonstrated that unmet basic social and functioning needs were the largest predictors of poor quality of life among individuals with severe mental disorder (Olweny, 1992). According to Patrick \& Erickson (1993), life has two dimensions: quality and quantity. Quantity of life is expressed in terms of hard biomedical data such as mortality rates or life expectancy. Quality of life refers to complex aspects of life that cannot be expressed by using only quantifiable indicators; it describes an ultimately subjective evaluation of life in general. It encompasses, though not only the subjective sense of well being but also objective indicators such as health status and external life situations.

Mental illness in the home can affect not only the quality of family life but also the health of the family members. A stressful emotional climate, anxieties and practical burdens, can have harmful effects a on the physical and mental health of both adults and children. Children as well as adults experience adverse effects on their health and this possibility has been studied extensively. An important consideration is that if children's mental and physical health is affected, with possible long-term consequences, then the social costs of having mentally ill people in the home may be very high (Rutter et. al., 1976). Mental disorders involve suffering. If the illness is chronic, the individual may have struggle with his or her identity (what kind of person am I?) and with alterations in lifestyle (Charmaz, 1995). Protracted or chronic illness also affects the rest of the family. Taking care of a chronically ill individual is demanding and stressful. The marital relationship may suffer (Woods \& Lewis 1995). Family patterns may have to be altered and may be highly constrained by the sick member.

One reason for serious illness tends to disrupt family life is that it precludes proper role functioning. For example, those who are physically or emotionally ill may be incapable of adequately fulfilling the roles of spouse, parents, or, breadwinner. The normal functioning of family life may give way to a focus on the ill person. In extreme cases, most activities of family members reflect the ill person's needs and limitations. Poor interpersonal relationships can be a 


\section{Quality of Life in Schizophrenia and Bipolar Affective Disorder}

factor in the onset of a mental disorder, and mental disorders adversely affect interpersonal relationships (Palisi and Canning 1983). In sum, there is a relationship between patterns of interaction and healthy. Illness, weather physical or mental, tends to be associated with disturbed interpersonal relationship. The relation between interaction and illness may be a vicious circle: bad interpersonal relationships being a factor in the onset of illness, and illness being a factor in causing disturbed relationships. The inadequate care of the mentally ill has been compounded by deinstitutionalization movement that began in the mid-1950s (Lamb 1998). At that point, in part because of the new drugs that were available, state and country hospitals began discharging a great many patients, some of whom had serious mental disorders. Unfortunately, the reality diverged sharply from the ideal, with the result that the movement added to the problem of inadequate care (Wright, Avirappattu, and Lafuze 1999).

\section{Research Related to Quality of Life}

Broadly, quality of life is a person's sense of wellbeing and satisfaction with his or her life circumstances as well as a person's health status and access to resources and opportunities. Unfortunately, the factors that influence quality of life in schizophrenia and affective disorder are not well known. One such factor consistently shown to be negatively associated with quality of life is psychiatric symptoms (Lambert \& Naber, 2004). However, due to wide variations in measurement strategies and definitions of quality of life, it has been difficult to identify which psychiatric symptoms are most strongly associated with poor quality of life in individuals with schizophrenia and affective disorders.

Quality of Life in Severe Mental Illness

There is need to focus to determine the factors associated with or affecting subjective quality of life in people with severe mental illness. Hansson (2006) stated that subjective quality of life in people with severe mental illness is only to a lesser extent related to external life conditions. Major determinants are psychopathology, especially symptoms of depression and anxiety, and aspects of social network. Personality related factors such as self esteem are also influential. Comparative studies have further shown that patients in community care settings have a better subjective quality of life than patients in hospital settings (Hansson, 2006). Evans et al. (2006) reported that objective life conditions were worse in the severe mental disorder group than in mentally healthy population. Severe mental illness population scored low on overall subjective and objective quality of life than group with no mental disorder. There are negative effects of distress/ clinical factors (psychological distress, anxiety, depressive and negative symptoms) on quality of life of psychiatric patients (Ritsner et al. 2003). There is increasing evidence which suggests that psychosocial or stress process is related rather than clinical factors more accurately predict quality of life. People with long-term psychiatric illness are so disabled and impaired that they are difficult to be placed in the community. In a study done by Young (2004) explored the impact of a residential home on the quality of life of people with long- term mental illness after their discharge from mental hospitals. This research adopted a combined research methodology and quality of life data were obtained from both quantitative and qualitative analysis. This research study has demonstrated that residential home care can lead to a better quality of life, 


\section{Quality of Life in Schizophrenia and Bipolar Affective Disorder}

including both subjective and objective quality of life of people with long- term psychiatric illness.

\section{Schizophrenia: Impact on Quality of Life}

Many researchers attempted to study poor quality of life in schizophrenia despite significant improvement with pharmacological treatment. In one such research done by Solanki et al. (2008) studied quality of life in patient with schizophrenia and determined influence of clinical factors and socio- demographic variables on quality of life of schizophrenia patients. Social relationship domain of quality of life was significantly negatively correlated with occupation with employed patients reporting better quality of life in this domain. Overall scores on positive and negative symptom scale (PANSS) was significantly negatively correlated with physical, psychological, social relationship domains and total quality of life. Sullivan and others (1992) also noted that long term psychiatric disorders such as schizophrenia are more vulnerable to stress, are more dependent, have greater deficits in living skills and have greater problems in employment and in relationship to their social environment. They also reported that quality of life of chronic psychiatric patients (heterogeneous groups including patients with schizophrenia, chronic affective disorders, personality disorders, substance abuse, etc.) is impoverished especially in the domains of housing conditions, family environment, social network, financial circumstances, safety and practical skills.

Several studies have confirmed poor quality of life in schizophrenia despite significant improvement with pharmacological treatment. Gee et al. (2003) addressed the dearth of qualitative research by conducting a small-scale qualitative exploration of the impact of schizophrenia on quality of life. Ten quality of life domains were identified as being important: (1) barriers placed on relationships; (2) reduced control of behaviors and actions; (3) loss of opportunity to fulfill occupational roles; (4) financial constraints on activities and plans; (5) subjective experience of psychotic symptoms; (6) side effects and attitudes to medication; (7) psychological responses to living with schizophrenia; (8) labelling and attitudes from others; (9) concerns for the future and (10) positive outcomes from experiences. Domains identified by participants encompassed a wide range of factors that may be expected to contribute generally to engaging in a positive quality of life. Participants identified that it was the loss of these things as a direct consequence of having schizophrenia that influenced their quality of life. It would appear that in the main, factors that are secondary to the experience of schizophrenia are of most importance to participants. The relationship of personal characteristics, objective indicators, and self-esteem to quality of life (QOL) was investigated (Kunikata et al., 2005). Depressive mood and uncooperativeness were negatively correlated with self-esteem, and self-esteem was positively correlated with QOL. Self-esteem was considered to directly affect quality of life, and depressive mood and uncooperativeness to affect quality of life via self-esteem. Findings suggest that, in order to improve the quality of life of schizophrenia patients, interventions to help alleviate psychiatric symptoms (e.g. depressive mood, uncooperativeness) must be utilized. Subjective quality of life and psychosocial functioning constitute important treatment outcomes 


\section{Quality of Life in Schizophrenia and Bipolar Affective Disorder}

in schizophrenia. Brissos et al., (2011) in their study mentioned that greater symptom severity and worse insight were significantly associated with worse functioning in all quality of life domains. Symptoms were more moderately correlated with quality of life, with no significant correlations between quality of life and positive symptoms and insight levels. Symptom severity may exert a greater influence on social functioning than on subjective quality of life; however, social functioning was not associated with subjective quality of life.

\section{Quality of Life in Bipolar Disorder}

The cyclical nature of bipolar disorder, with its many remissions and symptom rich periods of exacerbation, can affect an individual's physical, emotional, social and functional well-being and significantly impact their overall quality of life. In a study Vojta and colleagues (2001) hypothesized that patients with manic symptoms would report significantly lower quality of life than would patients who were euthymic. Patients with mania/hypomania did show significantly lower mental health scores than euthymic patients with depressed or mixed patients showing significantly poorer QOL again. An attempt for qualitative study was made by Michalak et al. (2006), identified themes in the quality of life influences of bipolar disorder. Respondents described a wide variety of factors that influenced quality of life including adverse effects of medications, occupation, and level of education, physical functioning, environment, health care factors, leisure activities, routine and sexuality. Overall, those individuals described having undergone several years of hardship and adjustment. Some of the factors mentioned (e.g. Independence, stigma, and disclosure, identity and spirituality) are not frequently examined in relation to quality of life, yet they appear to have a significant impact on people's ability to lead full lives in the context of bipolar disorder. Several studies have also found that quality of life is compromised in people with bipolar disorder, even during period of clinical remission. Cooke and colleagues (1996) in their study reported that patients with bipolar-II reported significantly poorer quality of life than bipolar-I in the areas of social functioning and mental health. WHO estimates, bipolar disorder was the $6^{\text {th }}$ leading cause of disability worldwide among young adults at the turn of the century. For e.g., if bipolar disorder develops in a woman at the age of 25, she may lose 9 years in life expectancy (because of cardiovascular and other medical problems), 14 years of productivity and 12 years of good health (US DHEW Medical Practice Project 1979). Disturbingly the lifetime suicide rates of patients with bipolar disorder (treated or not) may be as high as $15 \%$. Calabrese et al. (2003) reported that bipolar symptoms have been associated with significant functional impairment, often having a negative impact on the performance of work related, leisure, and interpersonal activities. Many studies have also found that quality of life is compromised in people with bipolar disorder, even during period of clinical remission [(Arnold et al. 2000).

Euthymic patients are not necessarily a symptomatic as many have mild sub- syndromal symptoms, and several studies demonstrated that even residual depressive symptoms can be strongly associated with impaired quality of life. The relationship between quality of life and hypomania is less well understood. Both mania and hypomania can be associated with 


\section{Quality of Life in Schizophrenia and Bipolar Affective Disorder}

substantial depressive symptomatology, either in the form of 'dysphoric mania/ hypomania' or when the patient experiences a mixed episode. This understanding led Vojta and colleagues (2001) to hypothesize that patients with manic symptoms would report significantly lower quality of life than would patients who were euthymic. Patients with mania/ hypomania did show significantly lower mental health scores than euthymic patients with depressed or mixed patients showing significantly poorer QOL again (Sierra et al., 2005). Gazalle et al., (2007) evaluated the influence of manic symptoms on quality of life in a sample of adult bipolar disorder (BD) patients. Das et al. (2005) recently found that patients who screened positive for bipolar disorder had lower mental and physical quality of life scores than those who screened negative. Interestingly, some studies have demonstrated lower levels of quality of life in bipolar depression in comparison with unipolar depression. Levels of depressive symptoms (Vojta et al., 2001; Yatham et al., 2004; Sierra et al., 2005), female gender and time undiagnosed (Gazalle et al., 2007), have been put forward as predictors of worse quality of life in bipolar disorder samples. The association between socio-demographic characteristics and quality of life was found for gender, age, education, and employment status and living arrangement (Caron et al., 2005).

Several quantitative studies have now examined the relationship between quality of life and bipolar disorder and have generally indicated that quality of life is markedly impaired in patients with bipolar disorder. However, little qualitative research has been conducted to better describe patients' own experiences of how bipolar disorder impacts upon life quality. Michalak et al., (2006) stated that clinical characteristics of the affected sample ranged widely between individuals who had been clinically stable for several years through to inpatients who were recovering from a severe episode of depression or mania. Findings indicated that there is a complex, multifaceted relationship between bipolar disorder and quality of life. Most of the affected individuals we interviewed reported that bipolar disorder had a profoundly negative effect upon their life quality, particularly in the areas of education, vocation, financial functioning, and social and intimate relationships.

\section{Co-morbidity with Schizophrenia and Bipolar Disorder and Quality of Life}

The clinical presentation of bipolar disorder is usually associated with psychological suffering, functional Impairment, interpersonal problems and substantial burden. Bipolar patients with higher scores for depression were reported to present lower scores for quality of life. Anxiety is a frequent complaint among patients with bipolar patients, and anxious co-morbidities are more prevalent among patients with bipolar disorder than among patients with unipolar depression. Anna et al. (2007) assessed the impact of anxiety co-morbidity on quality of life of patients with bipolar disorder. Anxiety co-morbidity in bipolar patients was associated with lower scores in all domains of quality of life. Current anxiety co-morbidity was also associated with lifetime alcohol abuse and dependence, rapid cycling, lifetime psychosis, number of suicide attempts, and a lower score in the Global Assessment of Functioning measure.

Co-morbidity of psychiatric disorders plays a major role in poorer quality of life in schizophrenia disorder. Bache, (2008) studied to determine the relationship between symptoms of obsessive- 


\section{Quality of Life in Schizophrenia and Bipolar Affective Disorder}

compulsive disorder (OCD) co-morbid with schizophrenia, and QOL and functioning. Functionality and social relationships domains in schizophrenia- OCD patients showed similar patterns with schizophrenia only patients. Severity of psychotic symptoms was correlated with functionality but not with total quality of life scores in schizophrenia- OCD patients. Beside, severity of OCD symptoms was related with social relationships domain of life quality in schizophrenia- OCD group patients.

\section{Comparison of Quality of Life in Bipolar Disorder and schizophrenia}

Long term psychiatric disorders such as schizophrenia, and bipolar disorder are more venerable to stress, are more dependent, have greater deficits in living skills and have greater problems in employment and in relationship to their social environment. (Sullivan \& colleagues, 1992). They also reported that quality of life of chronic psychiatric patients (heterogeneous groups including patients with schizophrenia, chronic affective disorders, personality disorders, substance abuse, etc.) improvised especially in the domains of housing conditions, family environment, social network, financial circumstances, safety and practical skills.

Disability has been noticed by many researchers in long standing disorders such as BPAD, Schizophrenia, and recurrent depressive disorder (RDD) in a study done by Tarrier and Turpin (1992) compared the inter-episode quality of life and disability in patients with severe mental illness (schizophrenia, bipolar disorder, RDD) in remission with or without co-morbid medical illness. Disability score in bipolar group was significantly more in 'social role' and in the RDD group it was more in 'home atmosphere' with chronic co-morbid medical illness. In another group without co-morbid chronic medical illness, the bipolar group has significantly more disability in 'overall behavior' and 'social role', and the RDD group had significantly more disability in 'assets and /or liabilities' and 'home atmosphere' domain. It becomes clear from the study that chronic medical illness does not cause a difference in the quality of life between the two disorders. Schizophrenia is classically described as a disease of exacerbations and remissions, patients seldom returning to their per-morbid level of functioning after each episode. Recent opinions, however, dispute the contention that patients with schizophrenia invariably have a deteriorating course of illness. Over their life-time the range of recovery varies greatly; as reasonable estimate is that 20 - $30 \%$ of patients continue to experience moderate symptoms and 40- 60\% remain significantly impaired throughout life (Sim et al., 2004). Increased negative symptoms were particularly correlated with dysfunctional performance at work and socially. The widely held belief about mood disorders is that these disorders are readily treated, and that once treated they require little or no intervention. But epidemiological studies of mood disorders suggest that there is significant chronicity. Atkinson et al. (1997) in their study characterized the quality of life of the three patient groups with chronic mental illness and evaluated differences in reported life quality among the three groups. The study groups consisted of chronically mentally ill patients with schizophrenia ( $n=69)$, bipolar disorder $(n=37)$ and MDD $(n=35)$. Subjects were administered the quality of life index, and comparisons of both objective and self report life quality indices were made among the three groups. Results showed that two groups with mood 


\section{Quality of Life in Schizophrenia and Bipolar Affective Disorder}

disorders reported significantly lower scores on quality of life index than the patients with schizophrenia. Moreover, the scores on the quality of life index for patients with schizophrenia were very similar to those of the comparison group of physically ill patients. The opposite trend emerged when groups were compared with respect to objective indicators of life quality. Schizophrenic patients experienced moiré objectively aversive life circumstances than either of the affectively disturbed groups.

Many researchers attempted to compare quality of life of bipolar disorder and schizophrenics patients with normal population. Among such studies, Rojas et al. (2008) evaluated those patients with bipolar disorder present worse mental health and quality of life than the general population. Davenport (2008) replicated similar results. Author explained that bipolar disorder patients who are in remission might still experience sub-syndromal or residual symptoms, which can lead to impairment and disability. They reported that bipolar disorder patients had consistently lower quality of life than general population with significant difference observed on the physical health, psychological health and social relationships domain. (Young, 2004; Davenport, 2008). Rojas et al. (2008) in his study evaluated the quality of life of patients suffering from bipolar disorder in comparison with the general population. It was discovered that patients with bipolar disorder present worse mental health than the general population; in addition, bipolar disorder patient have a poorer quality of life at a physical level. This could be due to a higher consumption of addictive substances such as alcohol and tobacco, the long- term secondary effects of the pharmacological treatment and a more sedentary way of life. The research also suggests that people with bipolar disorder who suffer a low quality of mental life are those who started to suffer the disease before 20 years old, who have been suffering it for a longer time, who suffer the II subtype of the disease, who are dependent on tobacco and who are suffering depressive symptoms at present. Furthermore, work has made clear that depressive symptoms (sadness, listlessness, tiredness, concentration difficulty, insomnia, poor appetite, etc.) have a higher impact on quality of life than manic symptoms (excessive self-esteem, lack of inhibition, verbosity, hyperactivity, increase of sexual appetite, etc.). depressive symptoms also produce more disability or negative repercussions for work, family and social life; this observation reflects the fact that manic symptoms are usually shorter in time and have a good response to medication, whereas depressive one are usually more difficult to eliminate (Arnold et al. 2000). In the past, the first goal of bipolar disorder treatment was the reduction of symptoms of mania or depression, rather than the recovery of social functioning. Recently, as a result of an increased emphasis on patient needs, the concept of quality of life has been brought into the treatment of physical illnesses. Klara et al., (2011) examined quality of life data in patients with bipolar disorder in clinical remission and to determine the extent of the effects of demographic and clinical data on quality of life in these patients.

\section{Predictors of Poor Quality of Life in Schizophrenia and Bipolar Disorder}

Schizophrenia is a severe and debilitating disorder, which affects general health, functioning, autonomy, subjective wellbeing, and life satisfaction of those who suffer from it. Despite 50 years of pharmacological and psychological intervention. Schizophrenia remains one of the top causes of 


\section{Quality of Life in Schizophrenia and Bipolar Affective Disorder}

disability in the world. Numerous studies on the correlates and predictors of quality of life in schizophrenic patients have been performed. Among many factors affecting objective quality of life, the importance of psychopathological symptoms especially negative and general psychopathology [(Addington et al. 2003), (Brown et al. 2000), ( Priebe et al. 2006)], duration of untreated psychosis (DUP), pre-morbid social adjustment ( Browm et al. 2000), social support and demographic variables (age, gender) have been emphasized. The subjective quality of life of schizophrenic patients is described as associated with depression, anxiety, negative, and rarely positive symptoms [(Bechdolf et al. 2003), (Sim et al. 2004)]. Other studies have related to the role of social support [(Caron et al. 2005)], DUP and stress related factors (Ritsner et al. 2006). Most previous studies on factors affecting quality of life were cross sectional, included patients with various durations of illness, and analyzed only one of the two dimensions of quality of life. Factors related to subjective quality of life in schizophrenia have been extensively reported. Brown et al. (1996) found that length of illness and the cumulative duration of hospitalization significantly correlated with SQOL. Law et al. (2005) reported that SQOL was more related to negative symptoms and only weakly related to, or independent of, positive symptoms.

Psychopathological symptoms are responsible for subjective and objective quality of life. Among psychopathological symptoms, negative symptoms seem to play the most important role in objective quality of life, especially for the social engagement/ withdrawal and independence performance domains. Socio-demographic factors put little influence on functioning in the majority of domains of quality of life. The influence of subjective quality of life increases with duration of post hospitalization period due to increase in negative symptoms over the period of time (Bechdolf et al., 2003). Sierra et al. (2005) assessed the quality of life in bipolar patients, and analyzed a set of clinical variables and socio-demographic data that could act as quality of life predictors. The study concluded that bipolar patients experience lower functioning and wellbeing even in the stable phase of the disorder. There is growing evidence that the deleterious impact depressive episodes and sub-threshold depressive symptoms in bipolar disorder extends to quality of life and functioning [(Vojta et al. 2001), Altshuler et al. 2002)]. Altshuler and colleagues (2006) found that sub-threshold depressive symptoms of bipolar disorder were significantly predictive of impaired role functioning- specifically, impairment in work, home functioning, and relationships (Yatham et al. 2000).

A study done by Awad et al. (2007) aimed to quantify the prevalence of misdiagnosed bipolar disorder among the depression population and evaluate the quality of life impact on misdiagnosis. The misdiagnosis in associated with poorer quality of life than major depressive disorder or diagnosed bipolar disorder, which are recognized as having a considerable impact on quality of life. In a naturalistic longitudinal study conducted by Ritsner et al., (2003) determined predictors of perceived quality of life and explored changes that occurred regarding quality of life among schizophrenics and bipolar patients. Poor quality of life was not a more severe problem for schizophrenic than for bipolar patients. Improved quality of life of schizophrenics was associated with reduced paranoid and distress (obsessive and somatization) symptoms and 
increased self- efficacy and self- esteem ratings. Individual changes in quality of life index scores among bipolar patients were associated with changes in depression, sensitivity, expressed emotion, and task- oriented coping scores.

\section{Quality of life of Caregivers}

Schizophrenia and bipolar disorder are an "unending burden" on the family. Caring for a person with chronic mental condition is more stressful than caring for a person with a physical disorder or disability. The effects on family carers are diverse and complex and there are many other factors which may exacerbate or ameliorate how carers react and feel as a result of their role. Caregiving is uniquely stressful event. Being a long-term caregiver has been found to be associated with a variety of negative outcomes. The consensus among studies examining physical and mental health outcomes is that a majority of caregivers report feeling physically and emotionally distressed (Anthony- Bergstone, Zarit \& Gatz, 1988; Brodaly \& Hadzi Pavlovie, 1990; Clipp \& George, 1990b). Several studies report higher levels of psychological morbidity among caregivers compared to no caregiving samples of the populations. Anthony- Bergstone and her colleagues (1988) noted the increased feeling of hostility, anger, and anxiety among caregivers as compared to the general population. Women caregivers' generally report higher levels of psychological distress than men (Anthoney- Bergstone et al., 1988). The studies have found that caregivers frequently meet diagnostic criteria for depression or other diagnosis. In one of the most comprehensive studies of this kind. Studies found prevalence rate for depression ranging from $31 \%$ for men to $46 \%$ for women. These studies provide strong evidence that caregivers are at risk of increased psychiatric symptoms and clinical disorders. Carers, who are women, and those who are spouse, have higher rates of psychological morbidity, perhaps because of the increased stresses inherent in being married to a person with schizophrenia or bipolar disorder (Jungburer, 2004).

Carers who cohabitate are more likely to be depressed or psychologically stressed than those living apart (Broadly \& Hadzi- Pavlovic, 1990; Harper \& Lund, 1990). The gender of the patient does not appear to affect carer well- being is made for the increased likelihood for behavioral disturbances in men (Broadty \& Hadzi- Pavlovic, 1990). There have been no consistent associations found between carer age and psychological distress, with higher levels having been reported in older carers and in younger supporters. However, such comparisons need no control for the effects of relationship in looking at age (Brodaty \& Hadzi-Pavlovic, 1990; Draper et al., 1992). The relationship between behavioral disturbances in patient and caregiver depression (Brodaly \& Hadzi Pavlovic's 1990). Haley \& Pardo, 1989, however revealed a significant correlation between depressive mood ratings in carers and demand behavior in patient, although disturbance problems (accusations, temper outbursts, bad language, and aggression) showed no association. Baumgarten et al., 1992) employed large numbers of subjects and used multivariate procedures to analyze this relationship and reported a strong positive relationship between caregiver depression and aspects of aspects of behavioral disturbances in patient. Numerous studies have demonstrated that increased psychological morbidity in carers ( Garfstrom \& 


\section{Quality of Life in Schizophrenia and Bipolar Affective Disorder}

Winbald, 1995; Brodaly \& Hadzi- Pavlovic, 1990). Depression levels and rates are also high (Rosenthal et al., 1993; Gallagher et al., 1989).

Carers have poorer physical health than non-carer controls, higher levels of chronic conditions, prescription medications and doctor visits (Haley 1989) and more physical symptoms and poorer self-rated health. Those with poor psychological health are even more likely to have physical morbidity (Brodaly \& Hadzi- Pavlovic, 1990; Vehars et al., 1999). They concluded that carers may be vulnerable to infectious disease. Other indicators of poorer physical health are increased such as service use hospitalization, physician visit, drug use, aggregate use of health services, and less healthy behavioral as reflected by use of alcohol, smoking, sleep patterns, eating behavior and nutrition. Further, pre-existing conditions such as hypertension are more likely to be exacerbated by the care giving role. In a Sudanese study conducted by Awadalla et al., (2005) assessed subjective quality of life of caregivers of schizophrenia, affective disorder and neurosis using WHOQOL- BREF. Schizophrenia caregivers had lower scores than others. Caregivers' socio- demographic variables were significantly associated with quality of life. Their quality of life was predicted by their impression of patients and state of health. Spouses of people with mental disorder experience various forms of objective and subjective burden. This should negatively affect their quality of life. Angermeyer et al., (2006) investigated the quality of life of spouses of people with schizophrenia, depression or anxiety disorders. When compared with the general population, the quality of life of the spouses of mentally ill people was lower in the domains 'psychological well-being' and 'social relationships'. There was a significant association between the patient's functional level and the spouse's quality of life. In addition to the stress spouses are exposed to during acute episodes of illness, spouses also experience chronic burdens in their everyday life which can severely affect their living situation and wellbeing: insecurity and ambivalence in the relationship with the patient, changes regarding intimacy and familiarity, shifts in role distribution, lack of opportunity for relaxing activities, sorrow and fears regarding the further course of the illness, impairment of health and financial strains (Jungbauer et al., 2004).

\section{CONCLUSION}

In conclusion we can say that mental illness in the home can affect not only the quality of family life but also the health of the family members. A stressful emotional climate, anxieties and practical burdens, can have harmful effects a on the physical and mental health of both adults and children. The role of family influences in causing and perpetuating the disorder of schizophrenia and bipolar affective disorder, therefore a need was felt to understand how actually the construct of expressed emotion and caregivers' burden operate in different cultures that is to say what components of expressed emotion and burden of care giving are determining relapse and poor quality of life in different cultural context. Patients with disorders such as schizophrenia and bipolar affective disorder are more likely to relapse when there is high expressed emotion present in their living environment. The stress from the remarks and attitudes of the family is overwhelming because they feel like the cause of the problems. The patient then falls into the cycle of relapse. The only way to escape this vortex for the family is to go through 


\section{Quality of Life in Schizophrenia and Bipolar Affective Disorder}

therapy together to prevent the relapse. But before that it becomes necessary to understand that what is the reason behind such attitude towards a family member who is mentally ill, what is the cause of burden and what all changes the caregivers' and the patients' quality of life come across.

\section{REFERENCES:}

Addington, J., Coldham, E.L., Jones, T., Addington, K.D. (2003) the first episode of psychosis: The experience of relatives. Acta Psychiatrica Scandinavica. 108, 4; 285- 289.

Altshuler LL, Gitlin MJ, Mintz J et al. (2002), Subsyndromal depression is associated with functional impairment in patients with bipolar disorder. Journal of Clinical Psychiatry 63(9):807-811.

Angermeyer, M.C., Kilian, R., Wilms, H.U. \& Wittmund, B. (2006) Quality of life of spouses of mentally ill people. International Journal of Social Psychiatry, 52, 278-285.

Anna, K., Benício, N., Ana, C.A., Keila, M. C., Fernando, K. G., Juliana,T., Sabrina, Correa da Costa, Aida, S. (2007). Anxiety Comorbidity and Quality of Life in Bipolar Disorder Patients. The Canadian Journal of Psychiatry, Vol 52, No 3, 175-181.

Anthoney- Bergstone, C., Zarit, S.H. \& Gatz, M. (1988) Symptoms of psychological distress among caregivers of Dementia patients. Psychology and Aging. 3, $245-48$.

Arnold, L.M., Witzeman, K.A., Swank, M.L., McElroy, S.L., Keck, P.E., (2000) Health-related quality of life using the SF-36 in patients with bipolar disorder compared with patients with chronic back pain and the general population. Journal of Affective Disorders.57:235-9

Atkinson M, Zibin S, Chuang H. (1997) Characterizing quality of life among patients with chronic mental illness: a critical examination of the self-report methodology. American Journal of Psychiatry.154:99-105

Awad George, Voruganti Lakshmi.(2007) The burden of schizophrenia on caregivers: a review. Pharmacoeconomics. 26(2): 149-162.

Awadalla, W.A., Ohaeri, J.U., Salih, A.A., Tawfiq, A.M. (2005) Subjective quality of life of family caregivers of community living Sudanese psychiatric patients. Social Psychiatry Epidemiology. 40; 755- 763.

Baungarten, M, Baltista, R.N., Infante- Rivard, C., Hanlet, J.A., Becker, R., \& Gauthier, S. (1992) The psychological and physical health of family members caring for a elderly person with dementia. Journal of Clinical Epidemology. 45, 1: 61- 70.

Bechdolf A, Maier S, Knost B, Wagner M, et al. (2003). A psychological early intervention program for the prepsychotic prodromal state. A case report. Nervenarzt . 74(5):436-9.

Brissos, S., Balanzá-Martinez, V., Dias, V.V., Carita, A.I., Figueira, M.L. (2011) Is personal and social functioning associated with subjective quality of life in schizophrenia patients living in the community? European Archives of Psychiatry Clinical Neurosciences.;261(7):509-17.

Broadly, H., \& Hadzi- Pavlovic, D. (1990) Psychological effects on carers living with persons with dementia. Australian and New Zealand Journal of Psychiatry. 24; 351- 361. 
Brown, S., \& Biirstwistle, J. (1998). People with schizophrenia and their families: Fifteen year outcome. British Journal of Psychiatry, 173, 139-144.

Browne S, Clarke M, Gervin M (2000) Determinants of quality of life at first presentation with schizophrenia. British Journal of Psychiatry; 176: 173-176.

Browne, G., Bramston, P. (1996) Quality of life in the families of young people with intellectual disabilities. Australian Journal of Mental Healtht Nursing. 5, 120-130.

Calabrese, J.R., Hirschfeld, R.M., Reed, M. (2003). Impact of bipolar disorder on a U.S. community sample. Journal of Clinical Psychiatry. 64, 425- 432.

Caron, J., Mercier, C., Diaz, P., and Martin, A. (2005) Socio-demographic and clinical predictors of quality of life in patients with schizophrenia or schizo-affective disorder. Psychiatry Research. 15, 137, 203-213.

Charmaz, K. (1995). The body, identity and self: adapting to impairment. Sociological Quarterly. 36(4): 657-80.

Clipp, E.C., \& George, L.K. (1990b) Caregivers needs and patterns of social support. Journal of Gerentology. 45; S102- S111.

Cooke, R.G., Robb, J.C., Young, L.T. (1996). Well being and functioning in patients with BPAD assessed using the MOS 20- item short form (SF- 20). Journal of Affective Disorder. 39, 93- 97.

Das, K., Olfson, M., Gameroff, M.J., Pilowsky, D.J., Blanco, C., Feder, A., Gross, R., Neria, Y., Lantigua, R., Shea, S. and Weissman, M.M. (2005), Screening for bipolar disorder in a primary care practice. Journal of the American Medical Association, 293, 956-963

Davenport, L. (2008). Patient depression predicts bipolar disorder caregiver burden. Acta Psychiatric Scand. 118: 49- 56.

Davenport, L. (2008). Quality of life reduced in euphoric bipolar disorder. Perspective in Psychiatric Care. 44, 275- 284.

Doyle, M., Flanagan, S., \& Browne, S. (1999). Subjective and external assessment of quality of life in schizophrenia: relationship to insight. Acta Psychiatrica Scandinavia. 99,6: 466472.

Draper, B. Poulos, R., Poulos, C.J. (1992) Risk for stress in elderlay caregivers. International Journal of Geritric Psychiatry. 11: 227- 231.

Evans, S., Banerjee, S., Leese, M., Huxiey, P. (2006). The impact of mental illness disorder and healthy population samples. Quality of Life Research. 16, 17- 29.

Gallagher, D., Rose, J., Rivera, P., Lovett, S. \& Thompson, L.W. (1989) Prevalence of depression in family caregivers. Gerentology. 29, 4; 449- 456.

Garfstrom, M., \& Winbald, B. (1995) Family burden in the care of the Demented and Non Demented elderly- a longitudinal study, Alzheimers Disease and Associated Disorders, 9,2; 78- 86.

Gazalle, F.K., Hallal, P.C., Andreazza, A.C., Frey, B.N., Anna, M.K.S., Weyne, F., Costa, C.C., Santin, A., Kapczinski, F. (2007) Manic symptoms and quality of life in bipolar disorder. Psychiatry Research .153; 33-38. 


\section{Quality of Life in Schizophrenia and Bipolar Affective Disorder}

Gee, L., Pearce, E., \& Jackson, M. (2003) Quality of life in schizophrenia: a grounded theory approach. Health Quality Life Outcomes. 1: 31.

Haley, W.E., \& Pardo, P.L. (1989) Group intervention for Dementia family caregivers: A longitudinal perspective. Gerontologist. 29, 4; 478- 480.

Harper , S., \& Lund, D.A. (1990) Wives, husbands and daughters caring for institutionalized and non- institutionalized dementia patients: Towards a model of caregivers burden. International Journal of Aging and Human Development. 30, 4; 241- 262.

Hasson- Ofayon, I., Karvetz, S., \& Roe, D. (2006). Insight into psychosis and quality of life. Comprehensive Psychiatry. 47(4): 265- 269.

Jungbauer, J., Wittmund, B., Dietrich, S. \& Angermeyer, M.C. (2004) The disregarded caregivers: subjective burden in spouses of schizophrenia patients. Schizophrenia Bulletin, 30, 665-675.

Klara Latalova, Jan Prasko, Tomas Diveky, Dana Kamaradova \& Hana Velartova (2011). Quality of life in patients with bipolar disorder - a comparison with schizophrenic patients and healthy controls. Psychiatria Danubina, Vol. 23, No.1, pp 21-26

Kunikata, H., Mino, Y., Nakajima, K. (2005) Quality of life of schizophrenic patients living in the community: the relationships with personal characteristics, objective indicators and self-esteem. Psychiatry Clinical Neurosciences. 59(2):163-9.

Lambert, M., Naber, D. (2004). Current issues in severe mental illness: overview of patient accapetability, functioning capacity and quality of llife. CNS Drugs. 18: 5-17.

Michelak, E.E., Yatham, L.N., Kolesar, S., Lam, R.W. (2006). Bipolar disorder and quality of life: a patient centered perspective. Quality of Life Research. 15, 25- 37.

Olweny, C.L.M., (1992) Quality of life in developing countries. Journal of Palliative care. 8, 2530.

Palisi, B.J. \& Canning, C. (1983). Urbanization and social psychological well- being: a crosscultural test of three theories. Social Psychological Quarterly, 24: 527- 43.

Patrick, O.L., \& Erickson , P. (1993) Health status and health policy; quality of life in health care evaluation and resource allocation. New York, Oxford University press.

Priebe, S., Roeder- Wanner, U.U., Kaiser, W. (2006) Quality of life in Ist admitted schizophrenic patients: a follow up study. Psychological Medicine. 30; 225- 230.

Ritsner, M., Kurs, R., Gibel, A., Hirschmann, S., Shinkarenko, E., Ratner, Y. (2003) Predictors of quality of life in major payshosis: a naturalistic follow- up study. Journal of Clinical Psychiatry. 64: 308- 315.

Rojas, L.G., Manuel, G., Jose, A.M., Veguilla, R., Dolores, J. (2008). Quality of life in bipolar patients: a comparison with a general population sample. Bipolar Disorder. 10, 5: 625634.

Rosenthal, C.J., Sulman, J., \& Marshall, V.W. (1993) Depressive symptoms in family caregivers of long-stay patients. The Gerentologist. 33, 2; 249- 257.

Rutter, M.L., Quinton, D., \& Yule, B.A. (1976). Family Pathology and Disorder in the Children. New York: Wiley. 


\section{Quality of Life in Schizophrenia and Bipolar Affective Disorder}

Sierra P, Livianos L, Rojo L. (2005) Quality of life for patients with bipolar disorder: relationship with clinical and demographic variables. Bipolar Disorders. 7: 159-165.

Sim, K., Mahendram, R., Siris, S.G., Heckers, S., and Chong, S.A. (2004) Subjective quality of life in first episode schizophrenia spectrum disorders with comorbid depression. Psychiatry Research. 15, 141-147.

Solanki RK, Singh P, Midha A, Chugh K. (2008) Schizophrenia: Impact on quality of life. Indian Journal of Psychiatry. 50:181-6.

Sullivan G, Wells KB, Leake B. (1992) Clinical factors associated with better quality of life in a seriously mentally ill population. Hospital Community Psychiatry. 43:794-802.

Tarrier, N., Turpin, G. (1992) Psychosocial factors, arousal and schizophrenia relapse: the psycho physiological data. British Journal of Psychiatry. 161: 3- 11.

Vedhara, K., Cox, N.K.M., Wilcock, G.K. (1999) Chronic stress in elderly carers of dementia patients and antibody response to influenza vaccination. Lancet, 3531; 627- 31.

Vojta, C., Kinosian, B., Glick, H., Altshuler, L. and Bauer, M.S. (2001) Self-reported quality of life across mood states in bipolar disorder. Comprehensive Psychiatry, 42, 190-195.

Woods, N.F., \& Lewis, F.M. (1995). Women with chronic illness: their views of their families' adaptation. Nursing Journal. 16, 135-48.

Wright, E.R., Avirappattu, R.G., \& Lafuze, J.E. (1999). The family experience of deinstitutionalization. Journal of Behavioral Health Service Research. 26, 289- 304.

Yatham, L.N., Lecrubier, Y., Fieve, R.R., Davis, K.H., Harris, S.D., and Krishnan, A.A. (2000) Quality of life in patients with bipolar I depression: data from 920 patients. Bipolar Disorders, 6, 379-385.

Young, K.W. (2004). Quality of life of people with long- term psychiatric illness living in residential home. Internaltonal Journal of Psychosocial Rehabilitation. 9(1), 133- 145. 